\title{
ON THE AXIOMS FOR A COOPERATIVE GAME WITHOUT SIDE PAYMENTS
}

\author{
R. E. STEARNS
}

Introduction. In 1960 a set of axioms for cooperative games without side payments was suggested by Aumann and Peleg [1] which provides a neat generalization of the classical von Neumann-Morgenstern $n$-person theory [5]. In subsequent papers [2], [3], and [4], many results parallel to the classical theory have been proven in this new framework. The purpose of this note is to re-examine the axioms in light of a specific six-person game and to suggest a small and reasonable change in these axioms that will make the theory even more satisfying. In particular, it will be shown that this game has no von Neumann-Morgenstern solution so if there is to be any hope of a general existence theorem for solutions, the axioms need to be strengthened to exclude this example.

Proposed definition. In this section, the modified axioms will be presented and these will be compared with the axioms in [1] and [2]. If $N$ is an $n$ element set, $E^{N}$ will represent an $n$-dimensional Euclidean space with coordinates indexed by elements in $N$. The components of payoff vector $x \in E^{N}$ will be indexed with superscripts $\left(x^{1}, \cdots, x^{n}\right)$ and $x \geqq s y$ for $S \in N$ will mean $x^{i} \geqq y^{i}$ for $i \in S$. Intuitively, $H$ will be the convex hull of the finite set of possible game outcomes and $v(S)$ will designate the set of payoff vectors $x$ for which the coalition $S$ can, in some sense, effectively obtain at least $x^{i}$ for each $i \in S$.

Definition. A game is a triple $(N, v, H)$, where $N$ is a finite set, $H$ is a compact polyhedral subset of $E^{N}$, and $v$ is a function that carries each subset $S$ of $N$ into a subset $v(S)$ of $E^{N}$ so that:

1. $v(S)$ is closed;

2. $v(S)$ is convex;

3. $v$ is a comprehensive, i.e. $x \in v(S)$ and $y \leqq s x$ imply $y \in v(S)$;

4. $v$ is super-additive, i.e. $S_{1} \cap S_{2}=\varnothing$ implies $v\left(S_{1} \cup S_{2}\right) \supset v\left(S_{1}\right)$ $\cap v\left(S_{2}\right)$;

5. $v$ is proper, i.e. $v(S) \neq \varnothing$ for all $S \subset N$;

6. $x \in v(N)$ if and only if $x \leqq y$ for some $y \in H$.

$N$ is called the set of players and $v$ is called the characteristic func1962 .

Received by the editors September 14, 1962 and, in revised form, December 3, 
tion. The intuitive justification of most of these axioms is given in [1] and [2]. Condition (5), the only one which is new here, says that the members of a coalition are guaranteed something just by the play of the game. The "game" in [1] is the same as our game, except that conditions (5) and (6) are replaced by:

$\left(5^{\prime}\right) v(\varnothing)=E^{N}$

(6') $H \subset v(N)$.

The only difference between the "ordinary game" of [2] and the game here is that the former satisfies ( $\left.5^{\prime}\right)$ instead of (5).

Observe that the axioms here are stronger than those in [1] and [2]. Conditions (5) and (3) imply (5'), and (6) implies (6'). The advantage of these new axioms is that they exclude some examples (given in the next section) which violate one's intuitive notions as to what a game should be and which have no von Neumann-Morgenstern solutions. The key consequence of the definition here is the following:

Lemma. If $G=(N, v, H)$ is a game and $S \subset N$ and $x \in E^{N}$ are such that $x \in v(S)$, then there is a point $y \in H$ such that $y \geqq s x$.

Proof. By (5), there is a point $x^{\prime} \in v(T)$, where $T=N-S$. Let $z \in E^{N}$ be the point such that $z={ }_{S} x$ and $z={ }_{T} x^{\prime}$. By (3), $z \in v(S)$ and $z \in v(T)$; hence, $z \in v(N)$ by (4). By (6), there is a $y \in H$ such that $y \geqq z={ }_{s} x$. Q.E.D.

This lemma says that if the members of a coalition can guarantee themselves certain minimal payoffs, there is some payoff vector in $H$ that actually gives them such payoffs. This cannot be proven from the weaker axioms.

Counter examples. A six-person game in the sense of [1] will be given and will be shown to have no solution. Then this example will be modified slightly to become an example of a seven-person ordinary game in the sense of [2] which has no solution. The reader who is unfamiliar with the solution concept is referred to [1-4]. Briefly, the basic definitions are as follows:

1. For $x, y \in E^{N}$ and $S \subset N ; x \operatorname{Dom}_{S} y$ if and only if $x \geqq_{S} y$ and $x \in v(S)$.

2. For $K \subset E^{N} ;$ Dom $K=\left\{y \in E^{N} \mid x\right.$ Doms $_{s}$ for some $x \in K$ and $S \neq \varnothing\}$.

3. $A=\left\{x \in H \mid x^{i} \geqq v_{i}\right.$ for all $\left.i \in S\right\}$, where $v_{i}$ is the number such that $v(\{i\})=\left\{x \in E^{N} \mid x^{i} \leqq v_{i}\right\}$.

4. $V$ is a solution to $G$ if and only if $V=A-\operatorname{Dom} V$.

EXAMPLE 1. Let 


$$
\begin{aligned}
& N=\{1,2,3,4,5,6\} \\
& v(S)= E^{N} \text { for } S=\{1,3,5\},\{1,2\},\{3,4\},\{5,6\},\{1,2,3,4\}, \\
&\{1,2,5,6\},\{3,4,5,6\}, \varnothing, \text { and } N, \\
& v(S)=\left\{x \in E^{N} \mid x^{i} \leqq 0 \text { for } i \in S\right\} \text { otherwise; } \\
& H=\text { convex hull of } c=(2,0,2,0,2,0), \\
& p_{1}=(0,2,1,1,2,2), \\
& p_{2}=(1,1,2,2,0,2), \text { and } \\
& \quad p_{3}=(2,2,0,2,1,1) ; \\
& G=(N, v, H) . \quad
\end{aligned}
$$

Note. For each of the three coalitions $\{1,2\},\{3,4\}$, and $\{5,6\}$, there is a point in $H$ for which the coalition effectively obtains a value of 2 for each of its members; yet there is no point in $H$ where these coalitions obtain these values simultaneously. This is a hopeless violation of super-additivity and the lemma of the last section; a violation which cannot be patched up by changing $v$ outside of $H$. Thus, this example falls outside the scope of the definition here.

Proof that $G$ has no solution.

1. Point $c$ is in the core because some member of each nonempty set for which $c$ is effective obtains his maximum for points in $H$.

2. Let $L_{i}$ be the line segment between $c$ and $p_{i}$. We see that $A-$ Dom $c=L_{1} \cup L_{2} \cup L_{3}$ because $H=A$ and $c$ dominates other points in $H$ via $\{1,3,5\}$.

3. We wish to prove that, if $V$ is a solution, then $L_{i} \cap V=\{c\}$. Observing that $G$ is symmetric under the permutation $\pi=(135)(246)$, we shall assume without loss of generality that there is a point $x \neq c$ such that $x \in L_{1} \cap V$. If $y \in V$ is a point such that $y$ Dom $p_{1}$, it is easily seen that $y \in L_{2}, y^{3}=2>x^{3}$ and $y^{4}>p_{1}^{4} \geqq x^{4}$. Thus, $y$ Dom $\{3,4\}$ which is impossible; hence, $p_{1} \in V$. Since $p_{1} \operatorname{Dom}_{\{5,6\}} z$ for $z$ in $L_{3}-\{c\}$, $V \cap L_{3}=\{c\}$. But this means that $p_{2}$ is undominated by $V$ and hence $p_{2} \in V$. But $p_{2} \operatorname{Dom}_{\{3,4\}} p_{1}$ and this is the final contradiction which proves (3).

4. (1) says that $c$ must be in any solution $V$ and (2) and (3) say that $c$ must be the only point in $V$; but we know that $\{c\}$ is not a solution.

Now for the example which satisfies the definition in [2]. It is obtained from Example 1 by adding a dummy player and changing the characteristic function outside of $H$; hence the previous proof essentially proves that this also has no solution. 
EXAMPLE 2.

$$
\begin{aligned}
& N=\{1,2,3,4,5,6,7\} \\
& v(S)= E^{N} \text { for } S=\{1,2\},\{3,4\},\{5,6\},\{1,2,3,4\},\{1,2,5,6\}, \\
&\{3,4,5,6\},\{1,2,3,4,5,6\}, \text { and } \varnothing . \\
& v(N)=\left\{x \in E^{N} \mid x \leqq y \text { for some } y \in H\right\} \\
& v(S)=\varnothing \text { otherwise; } \\
& H=\text { convex hull of } c=(2,0,2,0,2,0,0), \\
& p_{1}=(0,2,1,1,2,2,0), \\
& p_{2}=(1,1,2,2,0,2,0), \text { and } \\
& p_{3}=(2,2,0,2,1,1,0)
\end{aligned}
$$

Further observations. There are several observations to be made about the definition of a game given here.

1. The von Neumann-Morgenstern theory [5] can be made to fit this framework. Letting $\bar{v}$ be the classical characteristic function, let $v(S)=\left\{x \mid \sum_{S} \max \left[x^{i}, \bar{v}(\{i\})\right] \leqq \bar{v}(S)\right\}, H=\left\{x \mid \sum_{N} x^{i} \leqq \bar{v}(N)\right.$ and $x^{i} \geqq \bar{v}(\{i\})$ for $\left.i \in N\right\}$.

2. An alternative definition is to replace condition (5) by:

$\left(5^{\prime \prime}\right) v(\{i\}) \cap H \neq \varnothing$ for all $i \in N$.

Since $\left(5^{\prime \prime}\right)$ and (3) imply (5) this yields to an even stronger definition. For the purposes of a solution theory, however, the definitions are equivalent. More precisely, one can show that, given a game $G=(N, v, H)$, there is a $\bar{v}$ such that $G^{\prime}=(N, \bar{v}, H)$ is a game also satisfying $\left(5^{\prime \prime}\right)$ and such that $x \operatorname{Dom}_{S} y$ in $G$ for $x$ and $y$ in $H$ if and only if $x \operatorname{Dom}_{S} y$ in $G^{\prime}$.

3. Under the definition in this paper, the composition (as defined in [1]) of games is still a game.

4. The natural definition of an extended game is to take $(N, v, H)$ satisfying only conditions (1) to (5). It may be seen that Example 1 provides an unassailable example of an unsolvable extended game. It should be pointed out, however, that contrary to the classical theory, no significant role has yet been found for such extended games.

5. It is now known that conditions (1), (3), (4), (5), and (6) are all necessary if there is to be an existence theorem. In addition to the big question of existence, there is the question as to the importance of the convexity condition. 


\section{REFERENCES}

1. R. J. Aumann and B. Peleg, Von Neumann-Morgenstern solutions to cooperative games without side payments. Bull. Amer. Math. Soc. 66 (1960), 173-179.

2. R. J. Aumann, The core of a cooperative game without side payments, Trans. Amer. Math. Soc. 98 (1961), 539-552.

3. B. Peleg, Solutions to cooperative games without side payments, Trans. Amer. Math. Soc. 106 (1963), 280-292.

4. R. E. Stearns, Three-person cooperative games without side payments, Annals of Mathematics Studies, Princeton Univ. Press, Princeton, N. J. (to appear).

5. J. von Neumann and $\mathrm{O}$. Morgenstern, Theory of games and economic behavior, 3rd ed., Princeton Univ. Press, Princeton, N. J., 1953.

Princeton University 